\title{
A mitochondrial DNA duplication as a marker of skeletal muscle specific mutations in the mitochondrial genome
}

\author{
M Mancuso, C Vives-Bauza, M Filosto, R Marti, A Solano, J Montoya, J Gamez, S DiMauro, \\ A L Andreu
}

J Med Genet 2004;41:e73 (http://www.jmedgenet.com/cgi/content/full/41/6/e73). doi: 10.1136/jmg.2003.012278

M itochondrial myopathies are often associated with point mutations in mitochondrial DNA (mtDNA), and are usually maternally inherited. However, they may also present as an isolated myopathy, often with ptosis and ophthalmoparesis, ${ }^{1}$ or with isolated exercise intolerance. ${ }^{2}$ In eight patients with exercise intolerance described by us in $1999,{ }^{2}$ there was no evidence of maternal inheritance, muscle histochemistry showed cytochrome c oxidase (COX) positive ragged red fibres, biochemistry showed complex III deficiency, and molecular genetic analysis revealed different mutations in the mitochondrial cytochrome b gene (CYB). Several other patients with lifelong exercise intolerance harboured mutations in other mtDNA protein coding genes. ${ }^{3}$ Characteristically, these mutations are not maternally inherited and are abundant (more than 90\%) in skeletal muscle, where they coexist with smaller amounts of wild type mtDNA genomes. As a rule, the mutations are not present in other tissues and, surprisingly, are not found in cultured myoblasts, indicating that they are somatic mutations of skeletal muscle. However, the mechanism by which these mutations accumulate in muscle remains unknown.

As the clinical presentation of all patients (lifelong progression of clinical symptoms), suggested that the molecular defect may accumulate over time, we looked for duplications in the D-loop as putative sources of replication advantage or disadvantage for mutant mtDNAs. Here, we report our findings in 10 patients harbouring different mtDNA mutations restricted to skeletal muscle; 5 patients with point mutations and 5 with single deletions. In all five patients with point mutations, we identified a $652 \mathrm{bp}$ duplication in the mtDNA control region, which altered the promoter and replication elements. The duplication was restricted to skeletal muscle mtDNA, and analysis of subcloned PCR fragments encompassing both the site of the point mutation and the region of the duplication suggested that the duplication segregates independently from the point mutation. Our results suggest that the duplication is present only in the non-mutated molecules and confers a replicative disadvantage to wild type mtDNA, leading to the accumulation of mutated mtDNA molecules in skeletal muscle. Alternatively, this duplication may be a marker for the presence of skeletal muscle specific mutations in the mitochondrial genome.

\section{METHODS}

\section{Patients}

We studied 10 patients harbouring either pathogenic point mutations or single deletions restricted to skeletal muscle mtDNA. Five patients with lifelong exercise intolerance had point mutations: three in CYB (G15168A, G14846A, and G15084A), ${ }^{2}$ one in COXI (G5920A), ${ }^{4}$ and one in the tRNALeu $^{\text {CUN }}$ gene $(\mathrm{Gl} 2334 \mathrm{~A}) .^{5}$ Five patients had chronic progressive external ophthalmoplegia and myopathy associated with skeletal muscle restricted single deletions of

\section{Key points}

- We studied skeletal muscle DNA from ten patients (five with point mutations, and five with single deletions) in whom D-loop duplications were assessed by a back to back PCR strategy combined with subcloning experiments.

- We identified the same 652 nucleotide duplication in skeletal muscle from all five patients with point mutations. Moreover, the duplication was not present in a large series of control samples from human skeletal muscle. Subcloning analysis of PCR fragments encompassing both the duplication and the site of the point mutation suggested that each defect segregated independently.

- We propose that this duplication may be a marker for skeletal muscle restricted mutations in mtDNA and that it may play a key role in the accumulation of mutated mitochondrial DNA in these patients.

mtDNA. A large series of control DNAs from human skeletal muscle from our DNA bank (36 individuals) was included in the study to check for the presence of the duplication in normal population.

\section{Duplication assessment}

Because the amounts of tissues were insufficient for Southern blot analysis, duplications were assessed by a "back to back" PCR approach, which, with appropriate controls, has high sensitivity. The forward primer covered nucleotides 616-635, and the reverse covered nt 599-580. PCR conditions were those described by Wei et al: ${ }^{6}$ an initial cycle at $94^{\circ} \mathrm{C}$ for 3 minutes, $55^{\circ} \mathrm{C}$ for 3 minutes, and $72^{\circ} \mathrm{C}$ for 1 minute, followed by 30 cycles at $94^{\circ} \mathrm{C}$ for 40 seconds, $55^{\circ} \mathrm{C}$ for 40 seconds, and $72^{\circ} \mathrm{C}$ for 50 seconds. Furthermore, we coamplified a downstream fragment of $1201 \mathrm{bp}$ from an unaffected region of the mtDNA (forward primer, nt 14687-14706; reverse primer, nt 15887-15868), using blood DNA and a mixture of blood and muscle DNA for all patients, both as internal control and to rule out the possibility of amplifying a nuclear pseudogene.

The PCR products were subjected to electrophoresis through a $1 \%$ agarose gel and stained with ethidium bromide. The duplicated fragment was mapped by sequencing analysis of the amplified fragment. In two patients (those harbouring

Abbreviations: COX, cytochrome c oxidase; mtDNA, mtiochondrial DNA 
CYB mutations in G14846A and G15084A), the PCR product was subcloned and analysed. Briefly, PCR products were ligated into the pGEM-T vector and subcloned using the pGEM-T Easy vector system (Promega Corporation, Madison, WI, USA) in Escherichia coli JM109 cells. Cells were grown in selective medium, and individual colonies were cultured independently. Cloned plasmids were purified and sequenced by standard procedures. Fifty three clones for the G15846A patient and 48 clones for the G15084A patient were sequenced to exclude the presence of the duplication in the point mutated molecules.

To distinguish between the segregation of mutations and duplications, we performed PCR for the patients with CYB mutations followed by sequencing of an mtDNA fragment encompassing both the site of the CYB mutation and the segment of the control region that is duplicated. For these patients, a $2.9 \mathrm{~kb}$ PCR fragment was amplified (forward: nt 14687-15705, reverse: nt 1043-1024) with the following conditions: 10 cycles at $95^{\circ} \mathrm{C}$ for 2.5 minutes, $92^{\circ} \mathrm{C}$ for 20 seconds, and $68^{\circ} \mathrm{C}$ for 10 minutes, followed by 20 cycles at $92^{\circ} \mathrm{C}$ for 24 seconds, $68^{\circ} \mathrm{C}$ for 12 minutes, and $72^{\circ} \mathrm{C}$ for 10 minutes.

\section{RESULTS}

All five patients with skeletal muscle restricted point mutations also harboured the same $652 \mathrm{bp}$ duplication in the control region (fig 1). The duplication was present only in skeletal muscle DNA and it was absent in blood, where only the wild type band was detectable, ruling out the possibility that we had amplified a nuclear pseudogene (fig 1). In contrast, the duplication was absent in muscle mtDNA from all five patients harbouring single deletions. Mapping analysis showed that the duplication was flanked by a tandem repeat (СССССТСССС) at positions nt 305-314 and nt 956-965. By the same back to back PCR approach, we failed to identify other known duplications in the D-loop, suggesting a remarkable specificity for the 652 bp duplication.

Moreover, sequence analysis of PCR products encompassing both the area of the duplication and the site of the point mutation showed that mutated molecules did not harbour the duplication, suggesting that each defect segregated independently. Subcloning analysis of PCR fragments in two patients who harboured point mutations in the CYB gene failed to identify any duplicated molecule. In addition, the 652 bp duplication was not identified in a large series of 36 control skeletal muscle DNAs from our DNA bank, suggesting a remarkable specificity of the duplication for the skeletal muscle of patients with point mutations in their mitochondrial genome.

\section{DISCUSSION}

Tandem duplications of mtDNA regions have been associated with large scale deletions in patients with Kearns-Sayre syndrome, Pearson syndrome, and myopathy with diabetes.

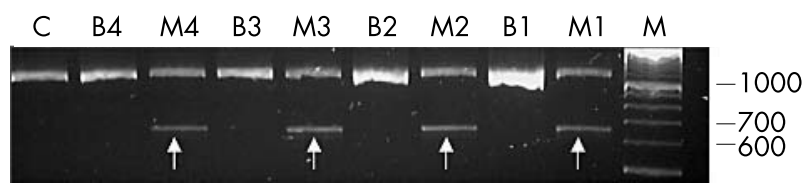

Figure 1 Identification of duplication in patients with mitochondrial myopathy. DNA from patients' muscle showed two bands, the wild type $1201 \mathrm{bp}$ mtDNA and a lower band corresponding to the $652 \mathrm{bp}$ duplication. DNA from the patients' blood did not have the $652 \mathrm{bp}$ band. $M 1 \rightarrow 4$, patients' muscle plus blood; $B 1 \rightarrow 4$, patients' blood; $C$, control muscle; $M$, molecular marker.
Duplications in mtDNA have also been described in healthy subjects $^{9}$ and as early molecular events in human ageing. ${ }^{6}$

Wei et al identified 10 types ( $\mathrm{I}-\mathrm{X}$ ) of tandem duplications in the mtDNA D-loop, defined by their location and sequence, ${ }^{6}$ and the $652 \mathrm{bp}$ duplication corresponds to Wei's type IV. Interestingly, all our patients were negative for duplications other than the $652 \mathrm{bp}$, and this was found only in patients harbouring point mutations restricted to skeletal muscle mtDNA (fig l).

To assess whether the duplication was associated with other pathogenic mtDNA mutations in muscle, we studied five patients harbouring single deletions restricted to muscle mtDNA; none harboured the duplication in their mitochondrial genomes. Moreover, an extensive study of the duplication in "normal" human skeletal muscle (36 individuals) failed to detect its presence, strongly suggesting that this duplication is highly specific for human muscle harbouring point mutations in the mitochondrial genome.

PCR analysis of fragments encompassing both the point mutation site and the duplication region showed that point mutations and duplication segregated independently. However, the coexistence of a muscle restricted $652 \mathrm{bp}$ duplication in pathogenic mutations restricted to the D-loop and muscle suggests that the two alterations may be related. A similar association was reported in the first patient with a CYB mutation, ${ }^{10}$ who harboured a 200 bp D-loop duplication. The authors suggested that the duplication might have resulted from free radical mediated single strand breaks in mtDNA secondary to respiratory chain dysfunction. ${ }^{10}$ However, the observation of an identical skeletal muscle restricted $652 \mathrm{bp}$ duplication in the control region of our patients suggests that the duplication may somehow be related to the high amounts of mutated genomes in these patients' muscle. A first hypothesis is that duplications in muscle with abundant mutations create a replicative advantage for the mutated molecules. However, analysis of PCR fragments encompassing both the point mutation and the duplication sites showed only the point mutation, suggesting that the duplication is not in the mutated but in the wild type molecule. Unfortunately, the structure of the duplication junction (CCCCCTCCCCCGC) makes it very difficult to design primers to assess whether the duplicated molecules also harbour the point mutation. However, subcloning results for the two patients harbouring $C Y B$ mutations support the idea that the duplication is present only in wild type molecules. The presence of a duplicated D-loop in the wild type mtDNA in these patients may have important consequences in terms of the accumulation of mutated mtDNA. We propose a scenario in which duplications and mutations do coexist during the first cycles of replication of mtDNA in myotubes. Wild type molecules would harbour the D-loop duplication, while point mutated molecules would harbour "normal" Dloops. Therefore, the accumulation of the point mutation in muscle would be due to replicative disadvantatge of the wild type DNA rather than replicative advantage of the point mutated genomes. This hypothesis would be consistent with the structure of the D-loop resulting from the duplication (fig 2). The $652 \mathrm{nt}$ duplication encompasses the conserved sequence boxes 2 and 3 (CSB2 and CSB3) and, notably, the replication primer sequence at the L-strand promoter. As the structure of this domain has to be conserved for replication to occur, the duplication may result in replicative dysfunction.

This hypothetical scenario is also consistent with the clinical presentation of patients harbouring skeletal muscle restricted mutations in mtDNA, because the course of the myopathy is slowly progressive, as expected if mutations accumulate gradually during successive replication cycles of mtDNA. However, we cannot exclude that duplications other than the $652 \mathrm{bp}$ seen in our patients might also imbalance 


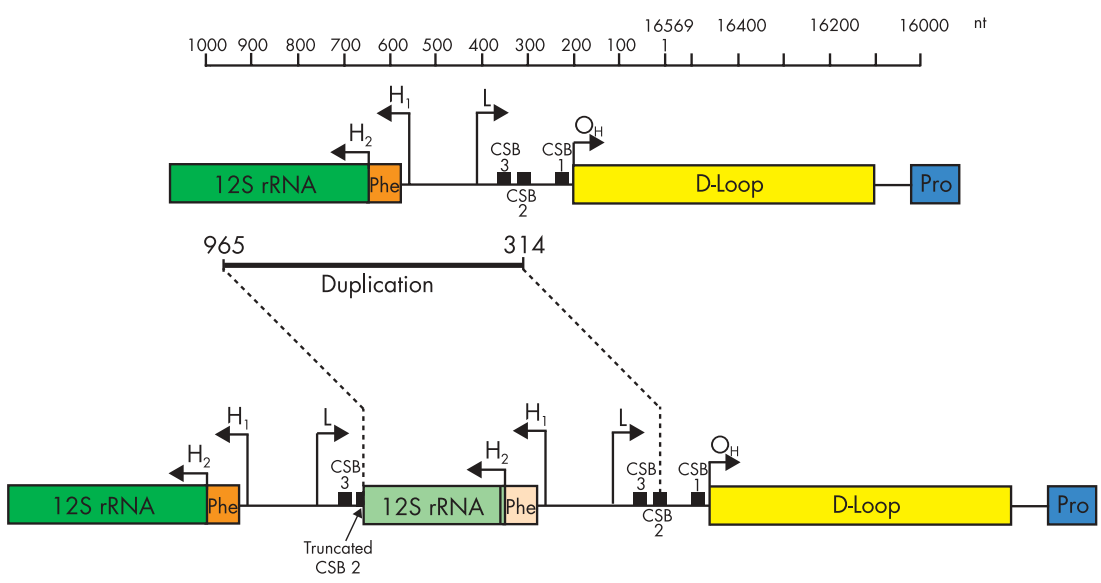

Figure 2 Structure of the duplicated region. $\mathrm{Hl}$, major $\mathrm{H}$-strand promoter; $\mathrm{H} 2$, minor $\mathrm{H}$-strand promoter; $\mathrm{L}, \mathrm{L}-$ strand promoter; $\mathrm{Oh}, \mathrm{H}$-strand origin of replication; CSB 1, 2, and 3, conserved sequence boxes 1,2 , and 3 . The rearrangement leads to a duplication of the promoters for the $\mathrm{H}$ - and $\mathrm{L}$-strands, whereas the major origin of replication is conserved. There is also complete duplication of the tRNAPhe gene and partial duplication of the 12S rRNA gene.

the replication rate of mtDNA in muscle. This could be true for the $200 \mathrm{bp}$ duplication identified in the patient harbouring the G15615A mutation. ${ }^{10}$

There is also an important consequence from a clinical research point of view. As the $652 \mathrm{bp}$ duplication seems to be remarkably specific for point mutations in skeletal muscle (it has not been detected in a large series of human skeletal muscles), it could be used as a rapid and inexpensive method for the screening of skeletal muscle when a mtDNA mutation is suspected. In conclusion, the presence of partially duplicated mtDNA genomes should be further studied to evaluate their role in the accumulation of mutant mtDNAs associated with lifelong exercise intolerance.

\section{ACKNOWLEDGEMENTS}

This study was supported by grants from the Spanish Fondo de Investigación Sanitaria (Network for Mitochondrial Disorders, grant no. G03-011) and from the FUNDISMUN Foundation, by NIH grants NS11766 and POIHD-32062, and by a grant from the Muscular Dystrophy Association. M Filosto was supported by the Departmen of Neurological Sciences and Vision, University of Verona, Italy, and M Mancuso was supported by the Department of Neurosciences, University of Pisa, Italy.

\section{Authors' affiliations}

M Mancuso, M Filosto, S DiMauro, Departments of Neurology, Columbia University College of Physicians and Surgeons, New York, NY, USA

A Solano, J Montoya, Departamento de Bioquimica y Biologia Molecular y Celular, Universidad de Zaragoza, Zaragoza, Spain C Vives-Bauza, R Marti, J Gamez, A L Andreu, Centre d'Investigacions en Bioquimica i Biologia Molecular, University Hospital Vall d'Hebron, Barcelona, Spain

The first two authors contributed equally to this work
Correspondence to: $\operatorname{Dr} A$ L Andreu, Centre d'Investigacions en Bioquimica i, Biología Molecular, Hospital General Vall d'Hebron, P. Vall d'Hebron 119-129, 08035 Barcelona, Spain; aandreu@vhebron.net

Received 26 September 2003

Accepted for publication 29 September 2003

\section{REFERENCES}

1 Di Mauro S, Bonilla E. Mitochondrial encephalomyophaties. In: Rosenberg RN, Prusiner SB, DiMauro S, Barchi RL, eds. The molecular and neurological basis of neurological disease, 2nd ed. Boston: Butterworth-Heinemann, 1997:201-35.

2 Andreu AL, Hanna MG, Reichmann H, Bruno C, Penn AS, Tanji K, Pallotti F, Iwata S, Bonilla E, Lach B, Morgan-Hughes J, DiMauro S. Exercise intolerance due to mutations in the cytochrome b gene of mitochondrial DNA. NEngl J Med 1999;341:1037-44.

3 DiMauro S, Schon EA. Mitochondrial respiratory-chain diseases. NEngl J Med 2003;348:2656-68.

4 Karadimas CL, Greenstein P, Sue CM, Joseph JT, Tanji K, Haller RG, Taivassalo T, Davidson MM, Shanske S, Bonilla E, DiMauro S. Recurrent myoglobinuria due to a nonsense mutation in the COXI gene of mitochondrial DNA. Neurology 2000;55:644-9.

5 Vives-Bauza C, Gamez J, Roig M, Briones P, Cervera C, Solano A, Montoya J, Andreu AL. Exercise intolerance resulting from a muscle-restricted mutation in the mitochondrial tRNALeu (CUN) gene. Ann Med 2001;33:493-6.

6 Wei YH, Pang CY, You BJ, Lee HC. Tandem duplications and large-scale deletions of mitochondrial DNA are early molecular events of human aging process. Ann NY Acad Sci 1996;786:82-101.

7 Poulton J, Holt IJ. Mitochondrial DNA: does more lead to less? Nat Genet 1994:8:313-15.

8 Lee HC, Pang CY, Hsu HS, Wei YH. Ageing-associated tandem duplications in the D-loop of mitochondrial DNA of human muscle. FEBS Lett 1994;354:79-83

9 Torroni A, Lott MT, Cabell MF, Chen YS, Lavergne L, Wallace DC. mtDNA and the origin of Caucasians: identification of ancient Caucasian-specific haplogroups, one of which is prone to a recurrent somatic duplication in the $D$ loop region. Am J Hum Genet 1994;55:760-76.

10 Bouzidi MF, Poyau A, Godinot C. Co-existence of high levels of a cytochrome b mutation and of a tandem $200 \mathrm{bp}$ duplication in the D-loop of muscle human mitochondrial DNA. Hum Mol Genet 1998;7:385-91. 